\title{
A98-37122 \\ MACE II: A SPACE SHUTTLE EXPERIMENT FOR INVESTIGATING ADAPTIVE CONTROL OF FLEXIBLE SPACECRAFT
}

\author{
Keith K. Denoyer* \\ Air Force Research Laboratory, Kirtland AFB, NM 87117 \\ David C. Hyland ${ }^{\dagger}$ \\ University of Michigan, Ann Arbor, MI 48109 \\ Lawrence D. Davis ${ }^{\ddagger}$ \\ Planning Systems, Inc., Melbourne, FL 32901 \\ David W. Miller ${ }^{* *}$ \\ Massachusetts Institute of Technology, Cambridge, MA 02139
}

\begin{abstract}
This paper presents an overview of the Middeck Active Control Experiment - Flight II (MACE II). MACE is a space shuttle flight experiment designed to investigate modeling and control issues for achieving high precision pointing and vibration control of future spacecraft. MACE was developed by NASA Langley Research Center, the Massachusetts Institute of Technology, and Payload Systems, Inc. The experiment was successfully flown on STS-67 in March 1995. The Air Force Research Laboratory (AFRL) has initiated a program to refly the MACE hardware to investigate the use of adaptive control algorithms for precision structural control. MACE II will answer key questions about the ability of adaptive algorithms to perform with respect to the constraints and uncertainties associated with space flight. It will also provide a basis for comparing these adaptive techniques with the fixed-gain linear control approach employed by MACE I.
\end{abstract}

* Dynamics Group Leader, Vehicle Technologies Branch, Space Vehicles Directorate, AFRL PRS/VSDV, 3550 Aberdeen Ave. SE, Kirtland AFB, NM 87117-5776.

$\dagger$ Chairman, Dept. of Aerospace Engineering, 3064 FXB Bldg., 1320 Beal St., University of Michigan, Ann Arbor, MI 48019-2118.

$\ddagger$ Controls Group Manager, Melbourne Controls Group, 1901 S. Harbor City Blvd., Suite 720, Melbourne, FL 32901.

** Assistant Professor, Dept. of Aerospace Engineering, 77 Massachusetts Ave., Space Engineering Research Center, Rm 37-387, Cambridge, MA 02139-4307.

1 This paper is declared a work of the U.S. Government and is not subject to copyright protection in the United States.

\section{INTRODUCTION}

The United States Air Force Research Laboratory (AFRL) is currently engaged in advancing technologies related to high precision deployed optical systems and other space systems requiring unprecedented structural stability and pointing accuracy. One example is the series of investigations known as the Ultra-Lightweight Imaging Technology Experiments (UltraLITE). These experiments are focused on demonstrating technological advances and examining integration issues related to large sparse optical array systems ${ }^{1-3}$. A concept for a deployed UltraLITE space-based imager is shown in Figure 1. Other applications of this technology include

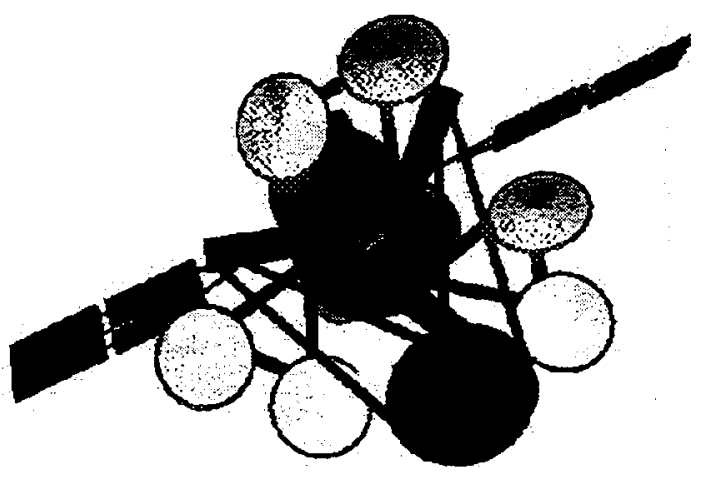

Figure 1. Concept for UltraLITE Golay-6

Space Based Laser (SBL) and Space Based Radar (SBR). An example concept for SBL is shown in Figure 2. Common features of many systems currently of interest are: large size $(>5 \mathrm{~m})$, extreme precision requirements (fractions of a wavelength of light), and a high 
 \\ Copyright $\odot 1998$, American Institute of Aeronautics and Astronautics, Inc.}

\section{.}

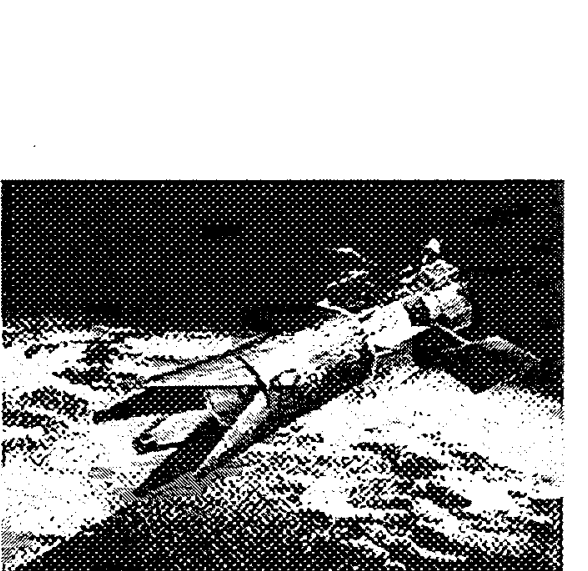

Figure 2. Space-Based Laser Concept

degree of system complexity (i.e. many sensors/actuators, layered controls loops, etc.). Because of the size, complexity, and precision requirements, on-orbit dynamics are expected to vary significantly from what is predicted by modeling and ground tests. Reliability, survivability and the ability to control the system in the presence of nonlinear behavior are also major concerns.

In recent years, we have witnessed a significant increase in the study of adaptive control methods. One reason is that adaptive methods address many of the difficulties encountered when traditional fixed-gain control is applied to complex spacecraft and other systems. Adaptive control has the potential to greatly reduce the resources required for modeling and control design, to recover autonomously from failures such as the loss of sensors or actuators, to adapt to dynamics which are time-varying or significantly different from those predicted from models and $1-\mathrm{g}$ ground tests, and (in the case of nonlinear adaptive control) to effectively control space systems exhibiting nonlinear behavior. This last issue is becoming increasingly important as the precision requirements of high performance space systems force us to consider sub-micron structural mechanics and other second order effects which may be nonlinear in nature. In addition, the capability to control nonlinear systems provides much greater flexibility to the spacecraft designers by allowing them to consider options that would otherwise be discounted simply because of our inability to model and control nonlinear behavior.

The Middeck Active Control Experiment (MACE) is a space shuttle flight experiment which flew on STS67 in March $1995^{4}$. The development of MACE was a joint effort between NASA Langley Research Center (NASA LaRC), the Massachusetts Institute of Technology (MIT), and Payload Systems, Inc. (PSI). The experiment is designed to investigate modeling and control issues needed to achieve high precision pointing and vibration control of future spacecraft systems. The MACE experiment is shown during operation on STS67 in Figure 3.

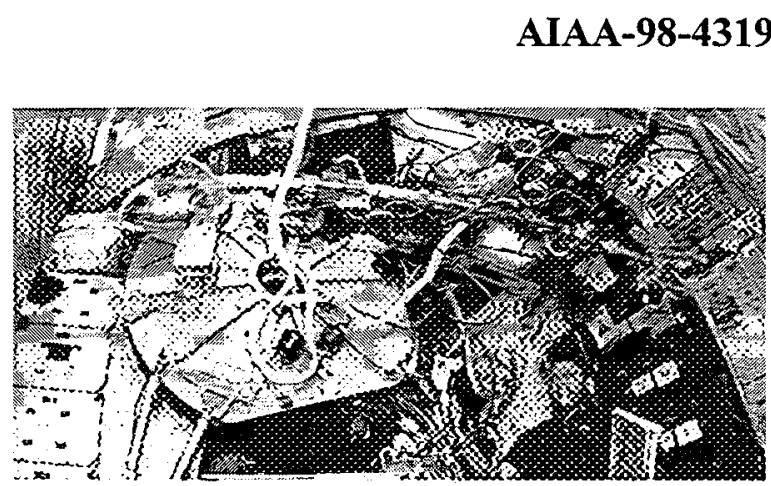

Figure 3. MACE in Operation During STS-67

The primary objective of the original MACE experiment was to demonstrate the effectiveness of structural control in improving spacecraft stability and to assess the predictability of controller performance based on analysis and $1-\mathrm{g}$ testing. To accomplish these objectives, a variety of techniques were developed to obtain accurate 0 -g models, along with associated parameter uncertainty models, using finite element modeling and $1-g$ ground testing. These models were then used to design a variety of fixed-gain control laws which were demonstrated on orbit and later modified during flight to improve performance and robustness using on-orbit data. The experiment was highly successful and demonstrated that structural control could be effectively accomplished using the deveioped techniques.

Despite these significant achievements, the MACE program also revealed limitations of the model-based fixed-gain linear control approach. These limitations include: significant expense and time associated with developing high fidelity finite element models needed for control design, loss of robustness due to unknown or unmodeled 0 -g dynamics, difficulties in handling nonlinear behavior, and the potential for loss of performance or instability due to time-varying dynamics or sudden failures of sensors and actuators.

To address these difficulties, there has been a significant interest in using adaptive methods for controlling structures in high precision aerospace applications. This is because adaptive methods offer the potential to autonomously adjust to system characteristics different from those modeled or seen in qualification testing. This is especially true of spacecraft, which are designed for 0 -g operation but must be tested in a $1-\mathrm{g}$ environment. Despite extensive research, MACE I and other experiments have shown that it remains extremely difficult to predict on-orbit $0-\mathrm{g}$ behavior. In addition, system dynamics often tend to be time varying. This can be due to thermal effects, slow changes due to degradation of materials and aging of the spacecraft, or sudden failures such as the loss of a sensor or actuator. These events become increasingly likely as spacecraft become more 
complex and are expected to be in service for longer periods of time. By decreasing modeling and testing requirements, lowering operations and maintenance activities, and increasing reliability and survivability, adaptive methods have the potential to significantly reduce cost and increase performance of these systems.

Because of these potential benefits, AFRL has conducted a series of programs to further develop adaptive control methods, particularly those that utilize artificial neural networks. The use of neural networks has become increasingly mature in a number of areas such as image processing and speech recognition. However, despite a number of publications on the subject, very few instances exist where neural networks have actually been used in control (particularly structural control) applications. One such application has been the demonstration of neural-network-based feedforward cancellation algorithm for rejecting multi-tone disturbances on the Air Force's ASTREX test article ${ }^{5,6}$. Other efforts are also currently underway to demonstrate adaptive neural structural control and other control approaches on the UltraLITE Phase I ground experiment ${ }^{7-9}$. Specific issues addressed include: developing more reliable methods for predicting convergence and performance of the algorithms, reducing the prohibitive computational burden needed to implement adaptive control, and familiarizing the community of potential users with adaptive methods.

As an extension of these efforts, AFRL is now conducting MACE II, which will be the first experiment to investigate and demonstrate adaptive structural control in a micro-gravity space environment. MACE II will answer key questions about the ability of adaptive control algorithms to maintain performance of a complex space system as its dynamics change from 1 -g qualification testing to $0-\mathrm{g}$. It will also demonstrate the capability to autonomously recover from subsystem failures such as the loss of an actuator or sensor. MACE II is being conducted by a diverse team that includes representation from government, industry, and academia.

\section{DESCRIPTION OF THE TEST ARTICLE}

The MACE II experiment will utilize the same hardware used in the original MACE experiment. The experiment consists of an approximately 1.5 meter flexible structure which can be reconfigured into various orientations. A schematic of the MACE test article is shown in Figure 4. Rigid body motion can be controlled by three reaction wheels located at the center of the bus and precision pointing is achieved using the two-axis gimbals located at the ends of the bus. A suite of various sensors and actuators exists for implementing a variety
AIAA-98-4319

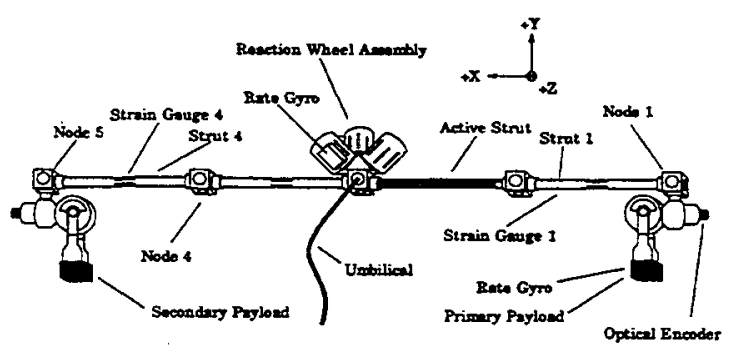

Figure 4. Schematic of MACE II Test Article

of control approaches. These include an active strut employing piezoelectric strain actuators.

The MACE structure is quite flexible, with the first bending mode of the MACE structure occurring at around $2 \mathrm{~Hz}$. Therefore, despite its small size it's behavior is similar to the behavior of large, lightweight structures. There are approximately 15 modes present in the $0-60 \mathrm{~Hz}$ control bandwidth.

The control processor for MACE II is the TMS320C30 DSP. The signal processing package will be capable of acquiring 1612 -bit $A / D$ inputs as well as 4 digital encoder inputs. The system is also capable of supplying up to 12 12-bit D/A outputs. The MACE software is being upgraded for MACE II to able to incorporate generic $\mathrm{C}$ code blocks for implementation of adaptive rather than fixed-gain controllers.

\section{MACE UOB.JECTUES}

The primary purpose of MACE II is to validate the ability of adaptive neural network-based and other adaptive algorithms to control, with little or no prior system knowledge, a representative small satellite system which alters its dynamics between 1-g qualification and $0-\mathrm{g}$ operation. Some specific objectives include:

1. Demonstrate that ground-achievable performance can be achieved on-orbit without the need for control redesign.

2. Demonstrate autonomous failure recovery to events such as sensor/actuator failure.

3. Demonstrate the savings that can be achieved by reducing modeling and testing currently required to achieve a high level of performance with fixed-gain non-adaptive controllers.

4. Demonstrate that adaptive algorithms can be implemented successfully using limited computational power consistent with space applications. 
5. Collect data for further evaluation of the ability of nonlinear modeling and identification tools to accurately predict $0-\mathrm{g}$ on-orbit behavior.

\section{CONTROL APPROACHES TO BE INVESTUGATED}

Several control approaches are currently under study for the MACE-II experiment. Final selection of control algorithms for the mission will be based on performance during simulation and ground test, as well as a demonstrated capability to meet the computational constraints of the MACE-II flight hardware. Some of the approaches under consideration are briefly described below.

\section{Adaptive Feedforward Cancellation}

Several linear and nonlinear adaptive feedforward cancellation approaches have been successfully demonstrated on test articles at AFRL and elsewhere. One example is the use of an adaptive neural controller to suppress a multi-tone disturbance on the AFRL's ASTREX test facility ${ }^{5,6}$. A photo of ASTREX is shown in Figure 5.

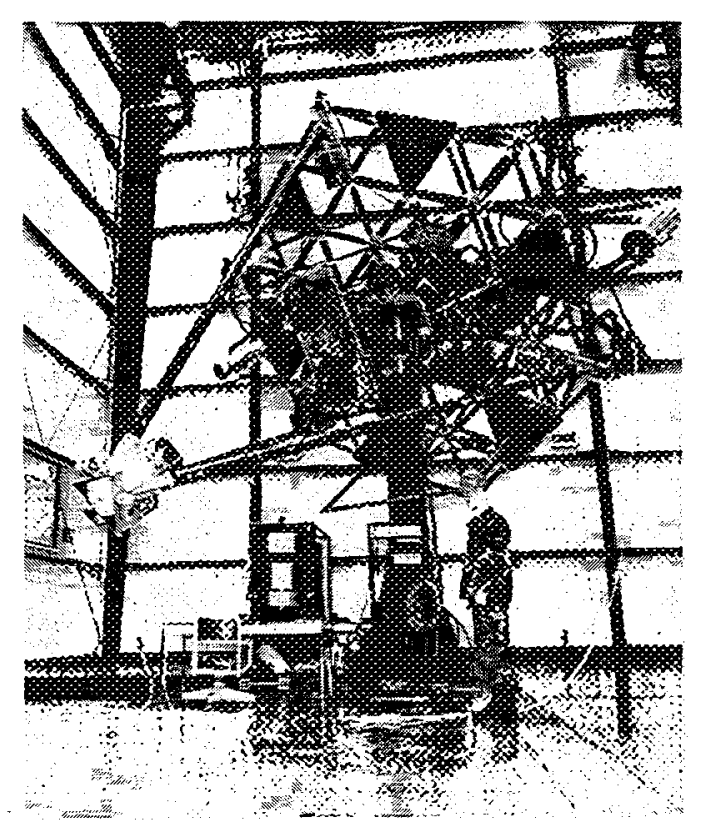

Figure 5. ASTREX Test Facility

In addition to this work, some recent efforts have focused on improving the convergence speed of the underlying neural-based system identifiers and extending this approach to the case of broadband control ${ }^{10-11}$. Current investigations are also looking at the possible use of these neural system identifiers in a feedback
AIAA-98-4319

architecture as well. Both SISO and MIMO controllers will be investigated for use on MACE II.

\section{Model Inversion Adaptive Neural Controller}

The objective for this controller is to exploit the information which is known well about the plant dynamics using a nominal model inverse-based controller and to correct for "inversion errors" using an adaptive neural network. In this sense, the approach can be viewed as a hybrid between traditional fixed-gain model-based control and adaptive neural control. The system dynamics are assumed to consist of a nominal linear component and an unknown varying perturbation:

$$
\begin{gathered}
\dot{x}=(A+\Delta A(x)) x+(B+\Delta B(x)) u+\Gamma w \\
y=C x+d
\end{gathered}
$$

where $x \in R^{n}$ is the state vector, $y \in R^{m}$ is the measurement vector, $u \in R^{m}$ is the control vector, $w \in R^{k}$ and $d \in R^{m}$ are uncorrelated, white noise disturbances. The matrices $\mathrm{A}, \mathrm{B}$, and $\mathrm{C}$ represent the nominal plant and $\Delta$ represents an unknown, but bounded, perturbation which can vary nonlinearly throughout the operating regime. The control objective is to use feedback to transform the known system dynamics into a desired form, which provides the appropriate transient and steady state behavior of the system.

\section{Adaptive Spatio-Temporal Filtering}

Adaptive Spatio-Temporal Filtering (STF) is a technique for separating the response of complex multiple degree-of-freedom (MDOF) system into a series of uncoupled single degree-of-freedom (SDOF) responses. By combining spatial filtering with past history time data, superior results can be achieved with limited sensor information. This technique has also been demonstrated experimentally at AFRL 9 .

Once the response is decoupled, the control problem becomes straight forward. One simply designs a Single Input Single Output (SISO) control law for each of the decoupled loops. An illustration of the approach in the frequency domain is shown in Figure 6 for control of the first two modes of vibration.

\section{Adaptive Positive Position Feedhack}

Positive Position Feedback (PPF) is a simple frequency domain design control approach that has been used extensively in smart structure applications ${ }^{12}$. Recently, the approach was also used successfully for suppression of both structural as well as acoustic cavity modes in a sub-scale test article representative of a launch vehicle fairing ${ }^{13}$.

An alternative to fixed-gain traditional PPF control is to use an adaptive PPF filter, which employs a Phase- 


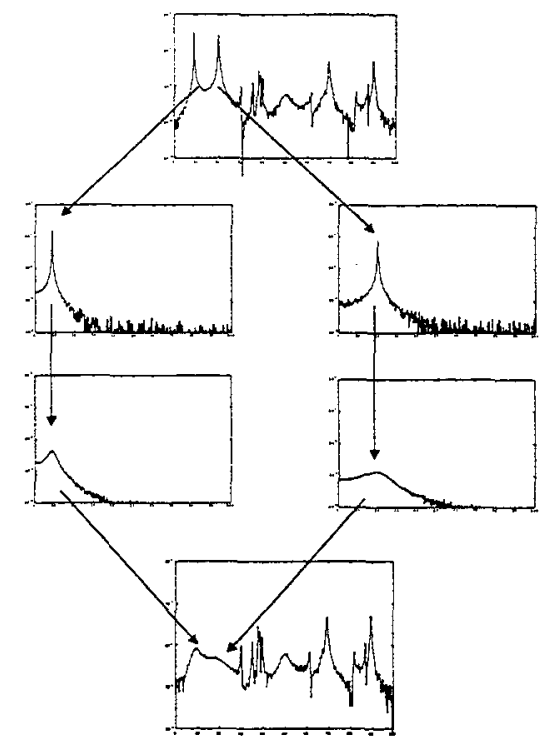

Figure 6. Illustration of Spatio-Temporal Filter Approach in Frequency Domain

Locked Loop (PLL) to track changes in frequency to the structural mode of interest that is to be suppressed. This has also recently been demonstrated in experiments at AFRL ${ }^{14}$.

In MACE-II, it is expected that localized active damping will be added to high amplitude modes of vibration using the piezoelectric strut. In addition to providing a nominal level of pointing performance, the addition of active damping is expected to improve the convergence speed of system identifiers in many of the adaptive algorithms that will be used for global pointing control. Difficulties associated with algorithm convergence in lightly damped systems are described in several papers ${ }^{10,11}$. While a simple fixed-gain active damping controller will probably be baselined, an adaptive local vibration suppression controller will also be examined. This controller will have the capability to provide a high level of vibration suppression, even when there are significant changes in the structural dynamics.

\section{CONCLUSIONS}

This paper provides an overview of the MACE II flight experiment. MACE II is a space shuttle experiment that will investigate several adaptive control approaches for precision structural control. In addition to providing a key demonstration that will help to transition adaptive control technology to operational systems, MACE II will provide key data for assessing the performance of these algorithms and comparing them to previously tested ap-
AIAA-98-4319

proaches. It is expected that MACE II will be extremely valuable in pinpointing areas where further research is needed.

Successful completion of experiments such as MACE II are viewed as critical in allowing adaptive control technology to go from state-of-the-art to stateof-the-practice. The benefit of this transition will be the availability of algorithms which have the ability to quickly and autonomously recover from subsystem failures, to be developed and maintained at greatly reduced cost, and to control systems which may be nonlinear in nature. It is expected that this research will allow complex future systems to have much greater robustness and reliability than possible with techniques currently used in spacecraft operations.

\section{ACKNOWLEDGMENTS}

The authors would like to gratefully acknowledge the collaboration and input received from the many partners associated with the AFRL Adaptive Structural Control Program. Special thanks go to Dr. Javier DeLuis from Payload Systems, Inc.; Dr. Stuart Shelley and Dr. Tom Sharp from Sheet Dynamics, Ltd.; Dr. Don Leo from the University of Toledo, and Dr. Jesse Leitner from AFRL.

\section{REEERENCES}

[1] Bell, K., Boucher, R., Vacek, R., and Hopkins, M., "Assessment of Large Aperture Lightweight Imaging Concepts," IEEE Aerospace Applications Conference, Snowmass, CO, Feb. 1996.

[2] Powers, M., Leitner, J., Bell, K., Boucher, R., Robertson, L., Schrader, K., and Hackney, E., "Assessment of a Large Aperture Telescope Trade Space and Active Opto-Mechanical Control Architecture," IEEE Aerospace Conference, Snowmass, CO, Feb. 1997.

[3] Robertson, L, Leitner, J., Slater, J., and de Blonk, B., "Integrated Modeling and Control of the UltraLITE System," IEEE Aerospace Conference, Snowmass, CO, Feb. 1997.

[4] Miller, D.W., Crawley, E.F., How, J.P., Liu, K., Campbell, M.E., Grocott, S.C.O., Glaese, R.M. and Tuttle, T.D., "The Middeck Active Control Experiment (MACE): Summary Report", MIT Space Engineering Research Center Report, SERC \#7-96, June 1996.

[5] Davis, L.D. and Hyland, D.C., "Adaptive Neural 
Control for Space Structure Vibration Suppression," Phillips Laboratory Technical Report, PL-TR-961133, August 1996.

[6] Hyland, D.C., Davis, L.D., Das, A., and Yen, G., "Autonomous Neural Control for Structure Vibration Suppression," AIAA Guidance, Navigation, and Control Conference, Paper No. AIAA-96-3923, San Diego, CA July 29-31, 1996.

[7] Denoyer, K. and Leitner, J., "Autonomous Neural Control for the UltraLITE Phase I Test Article," IEEE Aerospace Conference, Snowmass, CO, March 21-28, 1998.

[8] Denoyer, K. and Leitner, J., "Model Inversion Tracking Control for UltraLITE using Neural Networks," SPIE International Symposium on Optical Sciences, Engineering, and Instrumentation, San Diego, CA, July 19-24, 1998.

[9] Shelley, S., Sharp, T., and Denoyer, K. "Robust Line-of-Sight Stability and Jitter Compensationusing Spatio-Temporal Filter Based Approaches", SPIE International Symposium on Optical Sciences, Engineering, and Instrumentation, San Diego, CA, July 19-24, 1998.

[10] Hyland, D.C and Denoyer, K.K., "An Information Filter Approach to Rapid System Identification: Convergence Speed and Noise Sensitivity", Proceedings of the ALAA Guidance, Navigation, and Control Conference, Boston, MA, August 10-12, 1998.

[11] Hyland, D.C., Davis, L.D., and Denoyer, K.K."Accelerated Convergence of Neural Network System Identification Algorithms Via Principal Component Analysis", Proceedings of the ALAA Guidance, Navigation, and Control Conference, Boston, MA, August 10-12, 1998.

[12] Denoyer, K.K. and Kwak, M.K., "Dynamic Modeling and Vibration Suppression of a Slewing Structure Utilizing Piezoelectric Sensors and Actuators," Journal of Sound and Vibration, Vol. 189, No. 1, 1996, pp. 13-31.

[13] Denoyer, K.K., Griffin, S.F., and Sciulli, D., "Hybrid Structural/Acoustic Control of s Sub-Scale Payload Fairing," Proceedings of the 5th SPIE International Symposium on Smart Structures and Materials, March 1-6, 1998, San Diego, CA.
AIAA-98-4319

[14] Leo, D.J. and Griffin, S.F., "Microcontroller-Based Implementation of Adaptive Strucural Control," Proceedings of the 5th SPIE International Symposium on Smart Structures and Materials, March 1-6, 1998, San Diego, CA. 